\title{
Non-Linear pattern analysis of El Niño's impact on tropical precipitation
}

\author{
$X L \mathrm{Yu}^{1, *}$, and $S R$ Zhang ${ }^{1}$ \\ ${ }^{1}$ School of Science, Beijing University of Posts and Telecommunications, Beijing, China \\ ${ }^{2}$ School of Biological Science and Medical Engineering, Southeast University, Nanjing, Jiangsu, China
}

\begin{abstract}
Climate system is complicated and highly nonlinear, and a certain condition occurs over a small region could potentially propagate through space to affect remote regions. However, diagnosing such remote relationships is challenging due to the non-linearity of the climate system itself. In this study, we analyzed the relationship between Pacific Sea Surface Temperatures (SST) anomaly that indicates El Niño dynamics and Amazon basin local precipitation. We overcame the traditional difficulty of establishing reliable climate relationship with a simple linear assumption, by employing the statistical framework of mutual information. We effectively revealed a strong relationship between El Niño's and tropical precipitation as well as the spatial distribution of such relationship.
\end{abstract}

\section{INTRODUCTION}

As one of the most important and widely-acknowledged indicators of earth system climate variability, El Niño describes the Pacific basin-wide abnormal increase in both Sea Surface Temperatures (SST) in the central and eastern equatorial Pacific Ocean and in sea level and atmospheric pressure in the western Pacific (Southern Oscillation) [1]. It is also referred to as the phenomenon of El Niño-Southern Oscillation (ENSO) in the literature with the atmosphere and ocean collaborating together. El Niño in this context specifically ties to the warm phase of ENSO. ENSO exists because of the fact that the tropical Pacific features equatorward blowing trade winds under Coriolis force, accumulates an enormous volume of water above $28^{\circ} \mathrm{C}$ toward the Maritime Continent [2], which can be represented by tropical SST anomaly, and its temporal and spatial structures [3].

Although the phenomena of El Niño itself occurs over a relatively small region of tropical ocean, the impacts of El Niño can reach out to many remote regions of land surface and oceans due to the enormous changes in ocean and atmospheric circulations within and outside the tropical Pacific. With that, El Nino causes environmental disruptions such as devastating floods/droughts, coral bleaching events, destructive cyclone, and the associated abnormal warming can also affect natural ecosystem carbon cycle and greenhouse emission $[3,4]$. For instance, the 1997/1998 El Niño cost tens of thousands of human casualties worldwide and economic losses of tens of billions in U.S. dollars [5]. The impacts of El Niño could be even more dramatic during extreme El Niño events, which e.g., lead to significant social, economic, and environmental impacts [4]. El Niño can also cause the extreme rainfall reduction changes over land surface. For example, a long-term El Niño SST abnormity may give rise to rainfall reductions in Amazon basin [6]. In this study, we focused on analyzing the impact of the El Niño dynamics on Amazon basin local precipitation. One of the critical challenges of this study is to overcome the fact that previous studies drew conclusion of how El Niño affects regional climate based on the assumption of linear earth system coupling. We addressed this potential problem by employing a non-linear time series analysis approach that originally developed in information theory and applied to signal processing, called mutual information. Therefore, our object was to identify potentially hidden non-linear relationship and also spatial heterogeneity between El Niño dynamics and Amazon basin local precipitation, in contrast to a simple linear reconstruction of such relationship.

\section{Methodology}

\subsection{Data}

In this study, we employed Sea Surface Temperature (SST) anomaly over the region of longitude W-170 to 120 , and latitude -5 to 5 (so-called Nino3.4 index) to represent El Nino dynamics. Data ranges from 1958 to 2013 at a monthly resolution [7; Figure 1 upper panel]. We used precipitation data from Climate Research Unit (CRU) over the Amazon basin at $0.5 \times 0.5$ spatial resolution, and a monthly temporal resolution [8; Figure 1 lower panels]. Overall, Nino 3.4 index is a stationary time series across time but also includes several strong El Nino years, for instance, 1998 and 2009. Amazon basin receives a large amount of precipitation annually, in general. Spatially, there is also an apparent precipitation

Corresponding author: yuxinling@bupt.edu.cn 
gradient from northwest to southeast region, along which precipitation rate declines from over $4000 \mathrm{~mm} / \mathrm{yr}$ to $2000 \mathrm{~mm} / \mathrm{yr}$. However, variability of rainfall does not show apparent spatial trend, ranging from 50 to 200 $\mathrm{mm} / \mathrm{yr}$.

\subsection{Model}

Relationship between to climate variables can be established using linear correlation based on the assumption of linear relationship between those variables. In statistics, the Pearson correlation coefficient is a measure of the linear correlation between any two variables $\mathrm{X}$ and $\mathrm{Y}$. It has a value between +1 and -1 , where 1 is perfect positive correlation, 0 is no correlation, and -1 is perfect negative correlation. We first calculate the Pearson correlation between Nino3.4 time series and Amazon precipitation with:

$$
r=\frac{\sum_{i=1}^{n}\left(X_{i}-\bar{X}\right)\left(Y_{i}-\bar{Y}\right)}{\sqrt{\sum_{i=1}^{n}\left(X_{i}-\bar{X}\right)^{2}} \sqrt{\sum_{i=1}^{n}\left(Y_{i}-\bar{Y}\right)^{2}}}
$$

Where $\mathrm{Xi}, \mathrm{i}=[1,2 \ldots, 672]$ denotes Nino3.4 Yi, $\mathrm{i}=$ $[1,2, \ldots, 672]$ represents precipitation at each $0.5 \times 0.5$ resolution gridcell across the Amazon basin.

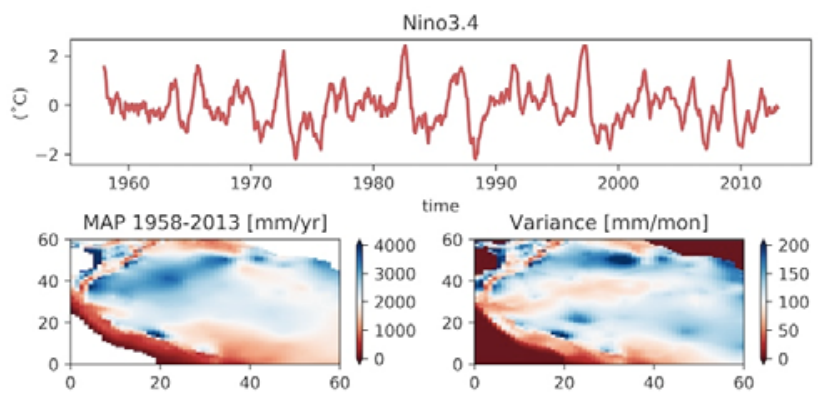

Fig. 1. Nino3.4 monthly time series from 1958 to 2013 (upper panel), Amazon basin mean annual precipitation (MAP) at $0.5 \times 0.5$ resolution (bottom left), and Amazon basin precipitation variance (bottom right).

We further relaxed the linear relationship assumption, which was probably not true for the complex climate dynamics and employed probability theory and information theory to use the mutual information (MI) of two random variables as a measure of the non-linear relationship. More specifically, it quantifies the "amount of information" (in units such as Shannon, more commonly called bits) obtained about one random variable, through the other random variable. The concept of mutual information is intricately linked to that of entropy of a random variable, a fundamental notion in information theory, which defines the "amount of information" held in a random variable. Here, we calculated the mutual information between Nino3.4 and Amazon precipitation to see their non-linear relationship. Let $H(X)$ be the Shannon entropy of random variable Amazon precipitation and $\mathrm{H}(\mathrm{X} \mid \mathrm{Y})$ be the conditional entropy of Amazon precipitation given Nino3.4. The mutual information $\mathrm{I}(\mathrm{X}, \mathrm{Y})$ is obtained as

$$
\begin{aligned}
& I(X, Y)=H(X)-H(X \mid Y) \\
& =-\sum_{x} p(x) \log _{2} p(x)+\sum_{y} \sum_{x} p(x, y) \log _{2} \frac{p(x, y)}{p(y)} \\
& =-\sum_{y} \sum_{x} p(x, y) \log _{2} p(x)+\sum_{y} \sum_{x} p(x, y) \log _{2} \frac{p(x, y)}{p(y)} \\
& =\sum_{y} \sum_{x} p(x, y) \log _{2} \frac{p(x, y)}{p(x) p(y)}
\end{aligned}
$$

where $\mathrm{p}(\mathrm{x})$ represents the probability density function (PDF) of random variable $\mathrm{X}, \mathrm{p}(\mathrm{y})$ represents the PDF of random variable $\mathrm{Y}$ and $\mathrm{p}(\mathrm{x}, \mathrm{y})$ represents the joint $\mathrm{PDF}$ of random variable $X$ and $Y$.

In our non-linear approach, it is critical to check the statistical significance of the mutual information, which means whether or not an identified relationship is significantly stronger than two independent time series. Here we used the method of shuffled surrogates. To estimate the shuffled mutual information, the values of $\mathrm{X}$ and $\mathrm{Y}$ were shuffled randomly aiming to remove time correlations between them and the new mutual information was computed through the shuffled time series and used as statistical threshold of our previous calculated mutual information.

\section{RESULTS}

\subsection{Linear coupling pattern analysis}

By assuming the system coupling between El Niño and Amazon local precipitation to be linear, we could reconstruct their relationship with the simple Pearson correlation coefficient. As shown in the Figure 2 left panel, we found that most of northern part of Amazon basin was negatively correlated with El Niño, while most of the southern part was positively related to El Niño. Moreover, neither showed a strong and significant correlation (absolute value of correlation $<0.3$ ). We expected that local precipitation should not be well related to remote El Niño dynamics due to spatial heterogeneity and local environmental conditions. Therefore, we also eliminated the spatial effect of regional heterogeneity, by integrating the precipitation over the whole Amazon basin and regressed with Nino3.4 (Figure 2 right panel). We found that the regional aggregated precipitation was negatively, but also weakly, correlated with El Niño dynamics.

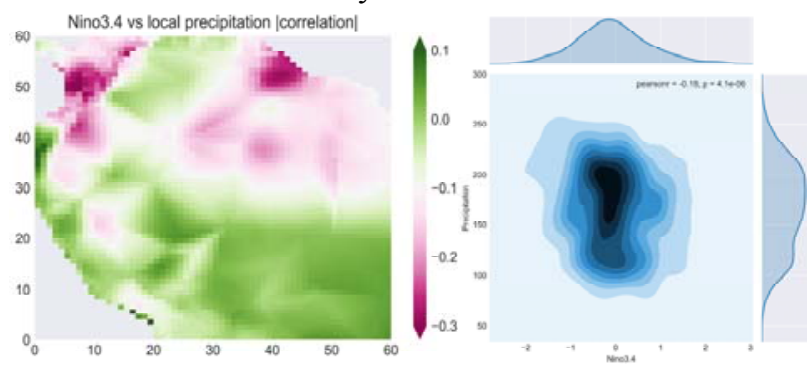

Fig. 2. Pearson correlation between Amazon basin local precipitation and remote Nino3.4 signal (left panel), probability density distributions and linear regression relationship of 
Nino3.4 versus average precipitation over the whole Amazon basin (right panel).

\subsection{Non-linear coupling pattern analysis}

We hypothesized that the atmospheric system was a highly non-linear system so that El Niño dynamics and remote local precipitation over Amazon basin should be non-linearly "correlated". Theoretically, mutual information (MI) between El Niño and the Amazon precipitation should be a better indicator of non-linear coupling than Pearson correlation. The Figure 3 illustrated the normalized mutual information that above the statistically significant threshold. A negative value (magenta) indicated no clear relationship, while a positive value (green) implied a strong relationship. Spatially, it showed an interesting spatial pattern that the heavy rainfall regime was less impacted by El Niño (Figure 1 and 3), while the relatively drier regions tended to have a strong non-linear coupling with El Niño dynamics.

\subsection{Limitation and future work}

Although we obtained a significant nonlinear relationship between Nino3.4 and Amazon basin precipitation under a nonlinear assumption that was more appropriate for climate system, mutual information limited our ability to retrieve the direction of this relationship (positive or negative relationship for example). This study was also limited to the temporal coverage of the most recent few decades, while our future study will continue to use nonlinear metrics to explore the coupling patterns between Amazon precipitation and climate anomaly under the future climate conditions with fully coupled earth system models.

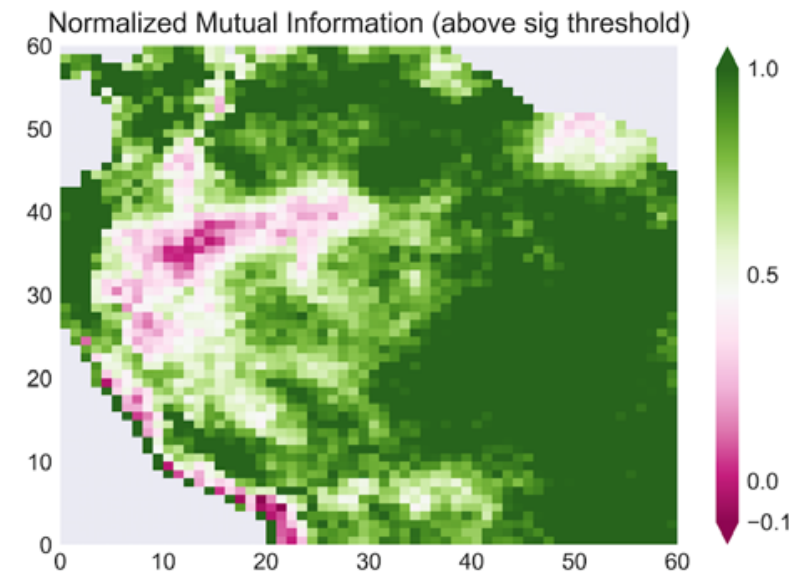

Fig. 3. Mutual information (MI: indicator of non-linear coupling) between Amazon basin local precipitation and remote Nino3.4 signal. MI is showed as values above its significant threshold, positive means strong coupling, and negative means no coupling.

Climate anomaly, such as El Niño, causes dramatic changes in atmospheric, oceanic and land surface changes including extreme regional weather. In this study, we focused on analyzing the relationship between the El Niño dynamics on Amazon basin local precipitation. We used two contrast methods to analyze the relationship with linear and non-linear assumption of climate system. The results showed that the linear relationship between El Niño and Amazon local precipitation was weak. However, our mutual information analysis successfully identified strong relationship between El Niño and Amazon local precipitation, which will potentially have a great implication on our understanding of El Niño dynamics and its potential remote impacts.

\section{References}

1. M. H. Glantz, Nature 385, 35 (1997)

2. S. Yang, Z. Li, J. Y. Yu, X. Hu, W. Dong, S. He, Natl. Sci. Rev. 5, 840 (2018)

3. A. Santoso, Rev. Geophys. 55, 1079 (2017)

4. G. D. Bell, J. Clim. 19, 590 (2006)

5. M. J. McPhaden, Science 314, 1740 (2006)

6. P. M. Cox, R. A. Betts, M. Collins, P. P. Harris, C. Huntingford, C. D. Jones, Theor. Appl. Climatol. 78, 137 (2004)

7. M. Luo, Y. Leung, Y. Zhou, W. Zhang, J. Clim. 28, 3122 (2015)

8. A. G. Pendergrass, D. L. Hartmann, J. Clim. 27, 8372 (2014) 